\title{
„Mit der Nadel einen Brunnen graben“
}

DOMiD wusste von Fehrs Überlegungen zum Migrationsmuseum. Aber einen wirklichen Austausch zwischen DOMiD und Fehr hat es nie gegeben, als die Vereinsvorstände Tayfun Demir, Lale Çakıroğlu, Ahmet Sezer oder Aytaç Eryılmaz Ende 1989 ihre Vision entwarfen und der Wirtschaftswissenschaftler Orhan Silier im Jahre 1990 auf einer Tagung in Gelsenkirchen eine mögliche Gründungsstrategie zum Aufbau eines solchen Museums systematisch durchdachte. Die Vision eines Migrationsmuseums stand also schon ganz am Anfang der Geschichte von DOMiD, auch in den Namen Dokumentationszentrum und Museum schreibt sie sich bis heute ein. Der Geschichts-Didaktiker Martin Schlutow stellt in seinem Buch zum Migrationsmuseum darum zu Recht die These auf, über die Sammlungsund Ausstellungspraxis eines Museums ließen sich Rückschlüsse auch „auf das Geschichtsbewusstsein der sozialen Träger eines Museums - der Gründungsväter, Sammler, Forscher und Ausstellungsgestalter“ ziehen. ${ }^{1}$ In einem der Gründungstexte aus DOMiDs Anfangszeit wurde die beabsichtigte Museumsgründung mit einem türkischen Sprichwort umschrieben: Die Schwierigkeit, Unwahrscheinlichkeit, ja beinahe Unmöglichkeit dieser Unternehmung sei dem Versuch vergleichbar, „mit der Nadel einen Brunnen zu graben“2. Gut zehn Jahre später schien das Migrationsmuseum dann erstmals tatsächlich in greifbare Nähe gerückt: Der nordrheinwestfälische Landtag forderte 2001 in einem Entschließungsantrag die Einrichtung eines Migrationsmuseums, und auch die SPD-Bundestagsfraktion diskutierte die Errichtung eines solchen Ortes. Diese günstige politische Debattenkonstellation veranlasste DOMiD dann im Oktober 2002, eine erste Tagung zum Aufbau eines Migrationsmuseums zu initiieren, um im darauffolgenden Jahr dann bereits konkrete Konzepte zu diskutieren. Auf der Folgetagung „Migrationsmuseum in Deutschland“, veranstaltet vom 17.-19. Oktober 2003 im Kölnischen Kunstverein, beklagte der Historiker Rainer Ohliger die fast „,vollkommene Abwesenheit einer kulturpolitischen Debatte“ über ein Migrationsmuseum in Deutschland und betonte die überragende kulturpolitische Bedeutung einer solchen Gründung. Gesellschaftlicher Zusammenhalt sollte nicht nur historisch-kulturell insinuiert, sondern zugleich auch politisch-institutionell begründet werden. Zumal Deutschland angesichts vergleichbarer Museumsgründungen im 
3 Rainer Ohliger, Kulturpolitik erweitern Migrationsgeschichte institutionalisieren: Herausforderungen für die Einwanderungsgesellschaft, Tagungsdokumentation, DoMiT 2004, S. $35 \mathrm{ff}$

europäischen Ausland endlich gleichziehen und plausible Antworten darauf finden müsse, was die Einwanderungsgesellschaft im Inneren zusammenhalte: „Wie kann ein erweitertes ,nationales kulturelles Gedächtnis ausgestaltet werden, das die engen Grenzen des Nationalen transzendiert, aber nicht notwendigerweise fragmentiert und parzelliert bzw. Migrationsgeschichte nicht allein im Randbereich der Gegengeschichte und der Geschichte des Kampfes um Anerkennung belässt, aber die Repräsentation der Migranten auch nicht harmonisierend in einer geglätteten Gesamtgeschichte Deutschlands (oder auch Europas) aufgehen lässt?“3

Ich habe damals selbst an der Tagung teilgenommen. Und ich erinnere mich, wie Rainer Ohliger Argumentationshilfen lieferte und gerade DOMiD - angesichts seiner Geschichte als Migrant ${ }^{\star}$ innenselbstorganisation eine besondere Legitimation zusprach, zum Träger eines solchen Migrationsmuseums zu werden. Indem der Historiker auf den wachsenden gesellschaftlichen Druck hinwies, eine solche Institution zu gründen, zumal im Hinblick auf uns - die zweite und dritte Generation der Einwander*innen-, suchte er selbst den nötigen politischen Handlungsdruck mit aufzubauen: „Das Interesse an der Errichtung von Migrationsmuseen bzw. der Musealisierung und Ausstellung der Migrationsgeschichte geht dabei in allen Fällen von einem bereits breiten Querschnitt von Fürsprechern aus Einwanderern, deren Kindern und Protagonisten der Mehrheitsgesellschaft aus. Zunehmend zeichnen sich dabei eine Lobby und auch ein Konsens ab, zunehmend vor allem mit der Sozialisierung einer zweiten und dritten Generation von Personen aus eingewanderten Familien in die etablierten Institutionen der Bundesrepublik. Dies verschafft der Initiative ein hohes $M a ß$ an Legitimität und Authentizität, die nicht gewährleistet wäre, wenn das Vorhaben allein als staatliche Unternehmung bzw. kulturpolitisches Vorhaben staatlicher Akteure ausgestaltet werden würde.."

Zur Umsetzung eines solchen Ortes hatte Aytaç Eryılmaz bereits während der Laufzeit des Projekts Migration, im Jahr 2003, den Verein Migrationsmuseum in Deutschland e.V. ins Leben gerufen. Darin sollten Vertreter ${ }^{\star}$ innen der verschiedenen Einwanderungscommunitys aus Italien und Spanien, Portugal oder Griechenland gemeinsam die Forderung nach einem Migrationsmuseum für Deutschland erheben - und dieser Forderung dadurch zusätzliches politisches Gewicht verleihen. 


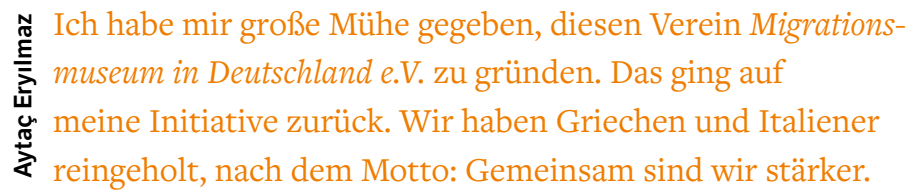

Dem Unterstützer*innenkreis gehörte Oscar Calero, Sozialberater der Caritas, für die Gruppe der Spanier*innen an; die Journalistin Paola FabbriLipsch repräsentierte die Italiener ${ }^{\star}$ innen, die Berliner Sozialberaterin Mira Renka stand für den Bereich ,Ex-Jugoslawien. Die Verlegerin Niki Eideneier vertrat den griechischen Bereich. Inter- bzw. transnationale Formen der Kooperation, wie sie in dem Verein Migrationsmuseum in Deutschland e.V. erprobt werden sollten, waren noch alles andere als selbstverständlich. DOMiD war von türkischen Intellektuellen gegründet worden, und lange wurde der Verein als, türkische Einrichtung wahrgenommen. Doch man hatte Hoffnung, diese Skepsis könne gemeinsam überwunden und eine Zusammenarbeit möglich gemacht werden.

5 VA 0528 (1) Protokoll Initiativkreis 18. Juni 2003.

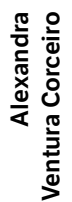

Das ist wirklich die große Leistung von DOMiD. Da ist eine Initiative aus der türkischen Community heraus entstanden. Und dann kommt einer wie Aytaç Eryllmaz 产 und sagt: „Wir müssen das gemeinsam machen! Das kann nicht nur die türkische Community machen, das wäre auch ein verzerrtes Bild.“

Im Jahre 2007 fand dann die Fusion DoMiTs mit dem Verein Migrationsmuseum in Deutschland e.V. statt. So groß die Hoffnung auf eine Art transnationale Lobbybildung für das deutsche Migrationsmuseum auch gewesen war - die Zusammenarbeit der Gruppen und Fraktionen innerhalb des Vereins Migrationsmuseum in Deutschland e.V. blieb kompliziert; der nun neu entstehende Verein DOMiD - Dokumentationszentrum und Museum über die Migration in Deutschland e.V. trug nicht länger das ,T‘ für ,Migration aus der Türkei' im Namen; stattdessen endete DOMiD zukünftig auf ,D“ wie ,Migration nach Deutschland'. So konnte DOMiD einerseits auf seine lange Vereinsgeschichte aufbauen, ohne sich weiterhin ausschließlich als eine Organisation türkischer Migrant*innen zu verstehen. Die Zusammensetzung seiner Vereinsorgane sollte die Vielfalt der Einwanderung nach Deutschland angemessen repräsentieren. So würde der Verein, so die Vision, in Zukunft Migrant*innen unterschiedlicher Herkunft sowie Deutsche ohne Migrationshintergrund gemeinsam unter dem Dach eines Migrationsmuseums für Deutschland vereinen. 
6 Aytaç Eryılmaz, Auf dem Weg zum Migrationsmuseum, in: Bundesamt für Migration und Flüchtlinge (Hg.): Deutsch als Zweitsprache, 2/2005, S. 36 ff., 0. O.

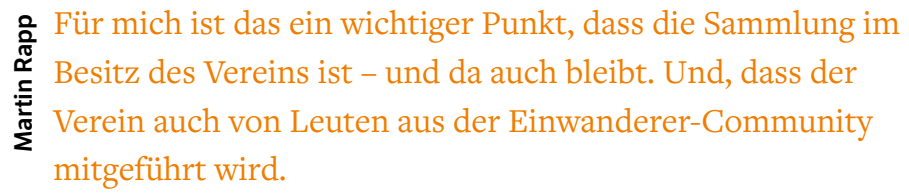

DOMiD festigte seine Rolle als anerkannter Experte in Fragen der Erinnerungskultur der Migrationsgesellschaft. Man könnte sagen: DOMiD wurde allmählich ,kanonisiert': 2005 erhielt man die Gelegenheit, in einem vom Bundesamt für Migration und Flüchtlinge herausgegebenen Band die Frage nach dem Migrationsmuseum aufzuwerfen. ${ }^{6} 2006$ wurde man zum Vorgespräch des 1. Integrationsgipfels ins Bundeskanzleramt nach Berlin geladen. Allerdings kamen Aytaç Eryılmaz bei diesem Treffen Bedenken, diesen politischen Prozess weiterhin zu begleiten:

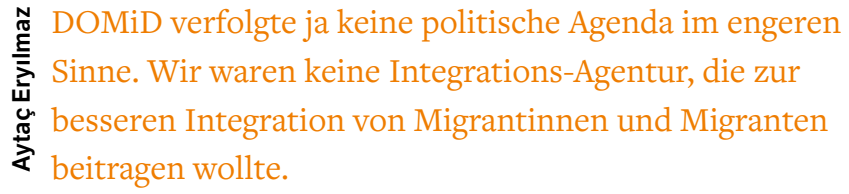

Auf internationalem Parkett wurde DOMiD allmählich zum anerkannten Akteur. 2005 reiste Aytaç Eryılmaz für einen Vortrag bei „Expert Meeting on Migration Museums“ von der Internationalen Organisation für Migration und der UNESCO nach Rom. Außerdem kooperierte man mit der Cité nationale de l'histoire de l'immigration in Paris.

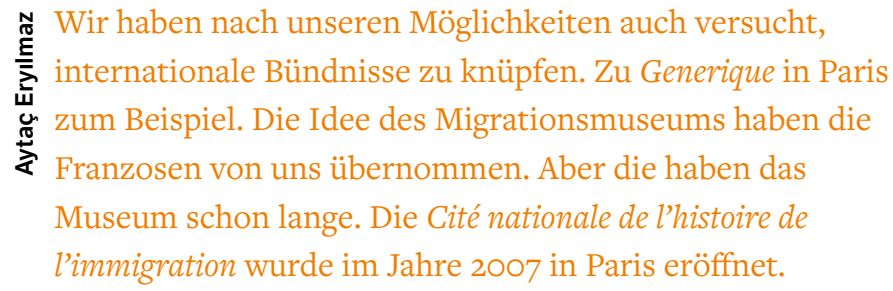


Auf der im Jahr 2009 von DOMiD in Kooperation mit dem Landschaftsverband Rheinland veranstalteten internationalen Fachtagung mit dem Titel Inventur Migration ging es buchstäblich darum, mit Fachkolleg*innen aus dem In- und Ausland eine Bestandsaufnahme zu machen, wie es um die Frage der Musealisierung der Migration in Deutschland und Europa bestellt sei. Dabei standen die Fragen „Welche Quellen zur Migration gibt es und wie wird diese Geschichte von Wanderungsbewegungen überlie-


Kriterien zur historischen Relevanz von Quellen zur Migrationsgeschichte erarbeitet - mit dem Ziel, ein Verzeichnis zur Migration zu erstellen, in dem alle Quellen und Objekte aus einer möglichst großen Bandbreite verschiedener Archive und Museen einfließen sollten. ${ }^{8}$

7 https://www.

land.nrw/de/

pressemitteilung/

minister-armin-lascheteroeffnet-internationalefachtagung-inventur-migration-wir (aufgerufen am 10.05.2021).

8 Eine Dokumentation der Tagung ist unter dem Titel „Inventur Migration“ erschienen. Köln 2009.

\author{
กี Ich habe immer zu Kollegen aus der Museumslandschaft \\ ह \\ 崖 \\ Abstimmung mit Leuten, die selbst Migrationserfahrung \\ oder Expertise haben. Dabei war mir eines besonders \\ wichtig: Wir wollten nicht nur ein ,Migrantenmuseum \\ gründen. Unser Horizont hatte sich erweitert. Unser Ziel war \\ es nun, gemeinsam mit anderen die Geschichte dieses Landes \\ zu erzählen.
}

